\title{
Caveats in BerEP4 staining to differentiate basal and squamous cell carcinoma
}

Background: Superficial skin biopsies of basal cell carcinoma (BCG) represent some of the most common dermatopathology specimens. Superficial shave biopsies containing partial samples of lesions with squamatization present difficulties in distinguishing BCC from squamous cell carcinoma (SCG). BerEP4 has been employed as a dependable marker in differentiating between BCGs and SCGs.

Methods: We collected 12 cases of superficial biopsies of BCC with centrally located cords and strands suggesting squamous differentiation at the Yale Dermatopathology Laboratory over a 3-month period and stained them with BerEP4.

Results: We found that all cases (12 out of 12) showed membranous and cytoplasmic staining with BerEP4 in the obvious areas of basaloid differentiation at the periphery of the tumors, while cords and strands of enlarged cells with squamoid features in the center and surface of the biopsy failed to label with BerEP4.

Conclusions: BerEP4 labeling is not reliable in superficial biopsies of BCG with squamoid features. It is important to be aware of this caveat in interpreting BerEP4 staining for BCG and SCG.

Yu L, Galan A, McNiffJM. Caveats in BerEP4 staining to differentiate basal and squamous cell carcinoma.

J Cutan Pathol 2009; 36: 1074-1076. (C) 2009 John Wiley \& Sons A/S.

\section{Limin $\mathbf{Y u}^{1}$, Anjela Galan ${ }^{2,3}$ and Jennifer M. McNiff ${ }^{2,3}$}

${ }^{1}$ Department of Pathology, University of Michigan Medical Center, Ann Arbor, MI, USA and

${ }^{2}$ Department of Dermatology and

${ }^{3}$ Department of Pathology, Yale University

School of Medicine, New Haven, CT, USA

Limin Yu, MD, M4211 Medical Science I, 1301 Catherine, SPC 5602, Ann Arbor, Ml 48109-5602, USA

Tel: (734) 7643270

Fax: (734) 7636476

e-mail: limin@med.umich.edu

Accepted for publication November 20, 2008
Superficial biopsies of basal cell carcinoma (BCG) represent some of the most common skin specimens received in pathology laboratories. With adequate biopsies, diagnosis is usually straightforward, based on peripheral palisading of basaloid nuclei, with cleft artifact between tumor islands and a specialized stroma. In some cases, however, superficial shave biopsies containing partial samples of lesions present difficulties in distinguishing BCG from squamous cell carcinoma (SCG), especially when only the surface of the lesion is visualized, and shows squamatization. This phenomenon seems most prevalent in small biopsies of ulcerated BCGs, in which the more obvious basaloid features expected at the periphery of the tumor are not contained in the biopsy. In such cases, small cords and strands of enlarged pale cells without basaloid features sometimes predominate. Moreover, this caveat seems under-recognized in major dermatopathology textbooks. ${ }^{1-3}$
In recent years, anti-human epithelial antigen (BerEP4) has been widely used as a reliable marker for BCC. Published data suggest that BerEP4 is a dependable marker in differentiating BCG from other neoplasms, most notably SCG, but also including actinic keratosis and microcystic adnexal carcinoma $(\mathrm{MAC}) .{ }^{4-7}$ In this study, we evaluated BerEP4 labeling in cases of BCGs with superficial squamous differentiation to evaluate its accuracy and reliability in distinguishing such cases from superficial samples of SCG.

\section{Materials and methods}

Twelve superficial biopsies of BCG with centrally located cords and strands suggesting squamous differentiation were retrieved from the histopathology archives at the Yale Dermatopathology Laboratory 
over a 3-month period. In addition, one case diagnosed as SCG during the study period, and identified as truly representing the surface of BCC on re-excision, was also included.

Serial recut sections of all cases were labeled BerEP4 monoclonal mouse anti-human antibody (DakoCytomation $\mathrm{GmbH}$ ) as the primary antibody, at a concentration of $1: 1000$ with an incubation time of $30 \mathrm{~min}$, followed by a biotinylated secondary antibody for $15 \mathrm{~min}$ and streptavidin peroxidase conjugate for another $15 \mathrm{~min}$. 3-Amino-9-ethylcarbazol was used to visualize the end product.

\section{Results}

Twelve cases were included. See Table 1 for clinical information. Twelve of 12 cases showed membranous and cytoplasmic staining with BerEP4 in the obvious areas of basaloid differentiation at the periphery of the tumors, while uninvolved epidermis was not stained. In each case, cords and strands of enlarged cells without basaloid features in the center and surface of the biopsy failed to label with BerEP4 (Fig. 1). In a biopsy of BCG that was originally interpreted as SCG prior to re-excision, the squamoid cords and strands present in the biopsy were negative for BerEP4. In contrast, the more clear-cut BCG on reexcision was strongly positive for BerEP4.

\section{Discussion}

BerEP4 reacts with 34-kDa and 39-kDa glycoproteins present on the cell surface and in the cytoplasm of most human epithelial and carcinoma cells. ${ }^{8}$ In the skin, BerEP4 stains normal adnexal epithelium, including the apocrine and eccrine epithelium, matrical and outer sheath epithelium of vellus anagen follicles and inferior segment epithelium of vellus telogen follicles. ${ }^{4,9}$ BerEP4 reacts with cutaneous BCG, Merkel cell carcinoma, trichoepithelioma, tumors with sweat duct differentiation and follicular

Table 1. Clinical information

\begin{tabular}{clllll}
\hline Case & Age (years) & Sex & Site & Clinical diagnosis & Size $\left(\mathrm{mm}^{3}\right)$ \\
\hline 1 & 70 & F & Left leg & AK, BCC & $5 \times 5 \times 2$ \\
2 & 50 & M & Left cheek & SCC & $4 \times 3 \times 1$ \\
3 & 79 & M & Forehead & SCC & $5 \times 2 \times 2$ \\
4 & 50 & F & Left chest & BCC & $6 \times 5 \times 1$ \\
5 & 71 & F & Nose & BCC & $5 \times 4 \times 1$ \\
6 & 53 & M & Nose & BCC & $5 \times 5 \times 1$ \\
7 & 59 & M & Face & SCC & $5 \times 4 \times 1$ \\
8 & 62 & F & Left leg & SCC & $2 \times 2 \times 2$ \\
9 & 78 & M & Left neck & BCC & $5 \times 5 \times 1$ \\
10 & 88 & F & Nose & BCC & $7 \times 5 \times 3$ \\
11 & 71 & M & Forehead & SCC & $5 \times 4 \times 1$ \\
12 & 61 & F & Nose & BCC & $8 \times 6 \times 1$ \\
\hline
\end{tabular}

AK, actinic keratosis; BCC, basal cell carcinoma; SCC, squamous cell carcinoma. induction over dermatofibromas. ${ }^{4,6,10}$ The usefulness of staining with BerEP4 to distinguish cutaneous BCC from SCC has been well shown. In a series with 22 cases of BCCs and 21 cases of SCGs, Tellechea et al. ${ }^{4}$ showed diffuse and intense labeling of BerEP4 in all BCGs, but no expression in any of SCGs. In another study performed by Kist et al., ${ }^{6} 27$ of 27 BCCs, including nodular, morpheaform/infiltrative and adenoid variants, were positive for BerEP4. BerEP4 defined areas of BCC in dense inflammation better than hematoxylin and eosin (H\&E) stain in 13 of 27 BCGs and identified the final Mohs margins in two cases of infiltrative BCC that were otherwise appearing negative with routine $\mathrm{H} \& \mathrm{E}$ stain. Our experience of BerEP4 immunoreactivity in SCG is consistent with the literature. All (5 of 5) SCGs retrieved from the same period as BCCs in this study were negative for BerEP4. It has also been shown that BerEP4 reliably differentiates MAC from BCC to the same extent as it distinguishes the latter tumor from SCG. ${ }^{5}$

BCC may show areas with morphologically distinct squamous cells, with or without keratinization. Those areas are usually located in superficial papillary dermis overlying more typical foci of palisaded epithelium and can make it challenging to diagnose BCG when only superficial biopsy is conducted. It is important to note that the clinical impression was 'SCC' in several of our cases, potentially misleading the pathologist in histologically ambiguous cases. To the best of our knowledge, no study has been done to evaluate BerEP4 expression in these cases. The data in this study suggest that staining for BerEP4 differentiate between BCG and SCG in such ambiguous cases is not reliable.

The terms 'basosquamous carcinoma' and 'metatypical carcinoma' are somewhat controversial and confusing but are most often applied to carcinomas with contiguous areas of both BCG and SCG, usually with less obvious peripheral palisading, and typically with an aggressive clinical behavior. Some authors feel that these tumors represent a transition between $\mathrm{BCC}$ and SCG. ${ }^{2}$ Keratotic BCG also shows squamous differentiation, typically in the form of keratin pearls and differentiation toward hair follicles. However, these features are usually located in the center of tumors, in contradistinction to the pattern observed in this report affecting the surface only and under areas of ulceration. Some basosquamous carcinomas also exhibit epithelial pearls. In one study, positive BerEP4 staining was identified in all basosquamous carcinomas, while two of the basosquamous carcinomas were negative for BerEP4 in small foci that had well-developed epithelial pearl formation. ${ }^{11}$

In summary, we commonly receive superficial biopsies in which the precise classification of carcinoma is problematic. While an attempt can be made to favor either BCC or SCG on histological grounds, this 


\section{Yu et al.}
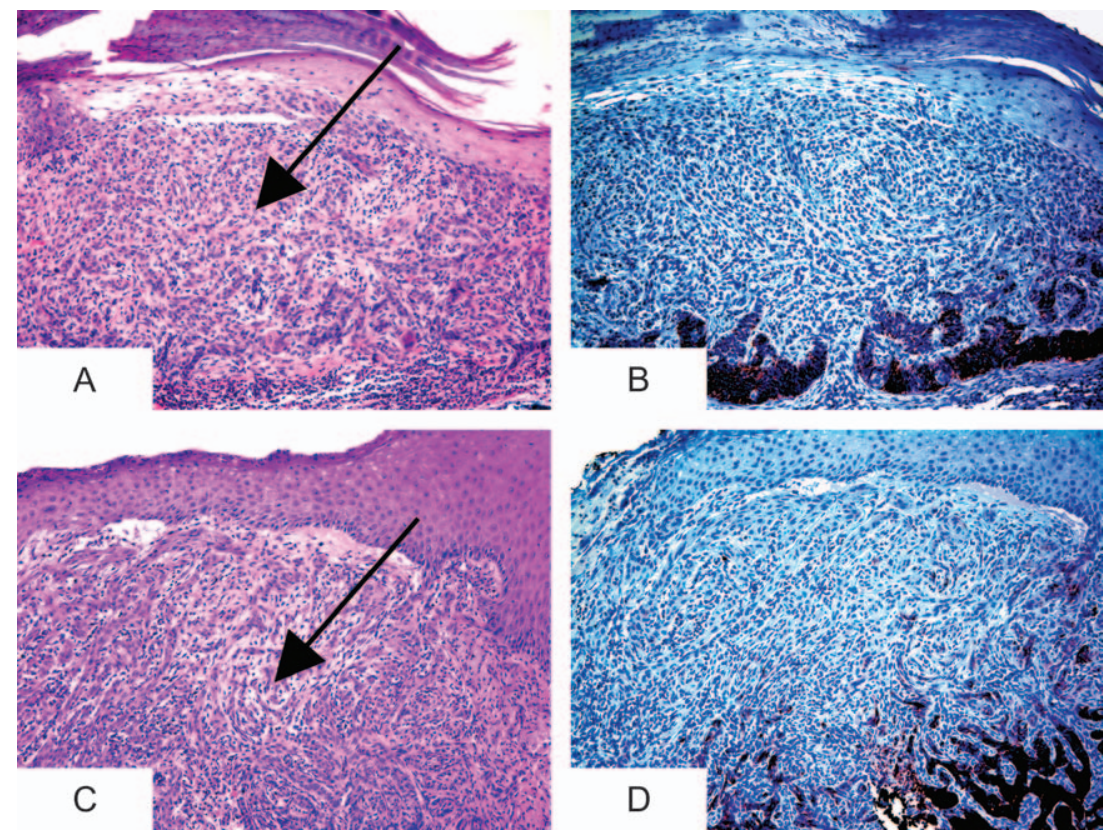

Fig. 1. BerEP4 labeled the obvious areas of basaloid differentiation at the periphery of superficial biopsies of basal cell carcinoma. Squamous differentiation in the center and surface of the biopsies (indicated by arrows) failed to label with BerEP4. A and B) H\&E and BerEP4 stains of one biopsy $(\times 400)$. C and D) H\&E and BerEP4 stains of another biopsy $(\times 400)$

is not completely reliable. We hoped, based on results in the literature, that staining with BerEP4 would be a useful adjunct in these cases. Unfortunately, despite strong staining of the periphery of such BCGs with BerEP4, staining with this antibody is consistently negative in superficial areas of tumor showing a more squamoid appearance. Awareness of this caveat in BerEP4 staining for BCG and SGG is important.

\section{References}

1. Barnhill R. Textbook of dermatopathology, 2nd ed. McGrawHill Professional, New York, NY, USA, 2004.

2. Elder DE, Elenitsas R, Johnson BL, Murphy GF. Lever's histopathology of the skin, 9th ed. Lippincott Williams \& Wilkins, Philadelphia, PA, USA, 2004.

3. Weedon D. Skin pathology, 2nd ed. Churchill Livingstone, Kidlington, Oxford, UK, 2002.

4. Tellechea O, Reis JP, Domingues JC, Baptista AP. Monoclonal antibody Ber EP4 distinguishes basal-cell carcinoma from squamous-cell carcinoma of the skin. Am J Dermatopathol 1993; 15: 452.
5. Krahl D, Sellheyer K. Monoclonal antibody Ber-EP4 reliably discriminates between microcystic adnexal carcinoma and basal cell carcinoma. J Cutan Pathol 2007; 34: 782.

6. Kist D, Perkins W, Christ S, Zachary CB. Anti-human epithelial antigen (Ber-EP4) helps define basal cell carcinoma masked by inflammation. Dermatol Surg 1997; 23: 1067.

7. Tope WD, Nowfar-Rad M, Kist DA. Ber-EP4-positive phenotype differentiates actinic keratosis from superficial basal cell carcinoma. Dermatol Surg 2000; 26: 415.

8. Momburg F, Moldenhauer G, Hammerling GJ, Moller P. Immunohistochemical study of the expression of a Mr 34,000 human epithelium-specific surface glycoprotein in normal and malignant tissues. Cancer Res 1987; 47: 2883.

9. Jimenez FJ, Burchette JL Jr, Grichnik JM, Hitchcock MG. Ber-EP4 immunoreactivity in normal skin and cutaneous neoplasms. Mod Pathol 1995; 8: 854.

10. Acebo E, Vidaurrazaga N, Varas G, Burgos-Bretones JJ, Diaz-Perez JL. Merkel cell carcinoma: a clinicopathological study of 11 cases. J Eur Acad Dermatol Venereol 2005; 19: 546.

11. Beer TW, Shepherd P, Theaker JM. Ber EP4 and epithelial membrane antigen aid distinction of basal cell, squamous cell and basosquamous carcinomas of the skin. Histopathology 2000; 37: 218. 